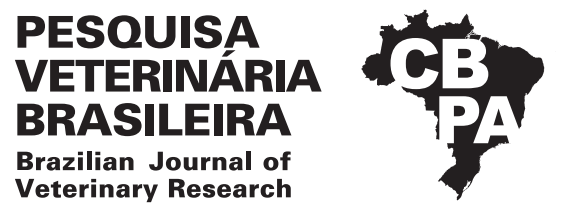

Pesq. Vet. Bras. 38(9):1767-1771, setembro 2018 DOI: 10.1590/1678-5150-PVB-5027

Artigo Original

Pequenos Animais/Small Animals Diseases

ISSN 0100-736X (Print)

ISSN 1678-5150 (Online)

\title{
Surto de esporotricose felina na região metropolitana do Recife $^{1}$
}

\author{
Grasiene M. Silva ${ }^{2 *}$, Julio Cesar F. Howes², Carlos Adriano S. Leal', \\ Emanuela P. Mesquita², Camila M. Pedrosa ${ }^{2}$, Andréia Alice F. Oliveira ${ }^{2}$, \\ Leonildo Bento G. Silva ${ }^{3}$ e Rinaldo A. Mota ${ }^{2}$
}

\begin{abstract}
Silva G.M., Howes J.C.F., Leal C.A.S., Mesquita E.P., Pedrosa C.M., Oliveira A.A.F., Silva L.B.G. \& Mota R.A. 2018. [Outbreak of feline sporotrichosis in the metropolitan area of Recife.] Surto de esporotricose felina na região metropolitana do Recife. Pesquisa Veterinária Brasileira 38(9):1767-1771. Departamento de Medicina Veterinária, Universidade Federal Rural de Pernambuco, Rua Dom Manuel de Medeiros s/n, Dois Irmãos, Recife, PE 52171-900, Brazil. E-mail: grasivet@hotmail.com

Sporotrichosis is a subcutaneous mycosis caused by a dimorphic fungus from Sporothrix schenckii complex, which affects various species, especially feline, able to cause local, disseminated or systemic impairment. We aimed to report the occurrence of an outbreak of feline sporotrichosis in the Metropolitan Region of Recife. Swabs of skin ulcers in cats treated in the Veterinary Hospital of the Federal Rural University of Pernambuco and in particular veterinary establishments were collected. Diagnosis was made by cytological examination and fungal culture. From 115 suspicious cases, 59 cases were confirmed, unprecedented results in the state of Pernambuco. These registered cases of feline sporotrichosis have demonstrated clinical and epidemiological characteristics similar to those occurring in other regions of the country, characterized as a quick-spreading disease, of difficult treatment, which affects predominantly semi-domiciled young males in reproductive age.
\end{abstract}

INDEX TERMS: Dimorphism, feline, cats, sporotrichosis, inoculation, ringworm, ulcers, Recife, fungus, mycoses.

RESUMO.- A esporotricose é uma micose subcutânea, causada por um fungo dimórfico do complexo Sporothrix schenckii que acomete várias espécies, especialmente a felina, causando comprometimento local, disseminado ou sistêmico. Objetivou-se relatar a ocorrência de um surto de esporotricose felina na Região Metropolitana do Recife. Foram coletados suabes de úlceras cutâneas de felinos atendidos no Hospital Veterinário da Universidade Federal Rural de Pernambuco e em Clínicas Veterinárias particulares. O diagnóstico foi realizado por meio de exame citológico e cultura fúngica. Dos 115 casos suspeitos, 59 casos foram confirmados, número nunca registrado anteriormente no estado de Pernambuco. Os casos de esporotricose felina registrados têm demonstrado aspectos clínicos e epidemiológicos semelhantes aos ocorridos

\footnotetext{
${ }^{1}$ Recebido em 14 de agosto de 2017.

Aceito para publicação em 21 de agosto de 2017.

${ }^{2}$ Departamento de Medicina Veterinária, Universidade Federal Rural de Pernambuco (UFRPE), Rua Dom Manuel de Medeiros s/n, Dois Irmãos, Recife, PE 52171-900, Brasil. *Autor para correspondência: grasivet@hotmail.com

${ }^{3}$ Departamento de Medicina Veterinária, Universidade Federal do Pará (UFPA), Rua Augusto Corrêa 1, Guamá, Belém, PA 66075-110, Brasil.
}

em outras regiões do país, caracterizando-se como uma doença predominantemente de machos jovens em idade reprodutiva e semi-domiciliados, de difícil tratamento e de rápida disseminação.

TERMOS DE INDEXAÇÃO: Dimorfismo, esporotricose, felinos, inoculação, micose, úlceras, Recife, fungo, micoses.

\section{INTRODUÇÃO}

A esporotricose é uma micose subcutânea de caráter subagudo ou crônico causada por um fungo do complexo Sporothrix schenckii. 0 primeiro caso de esporotricose foi registrado por Benjamin Schenck, em 1898 nos Estados Unidos e desde então foram descritos episódios isolados e surtos em vários países (Barros et al. 2010). No Brasil, Lutz \& Splendore (1907) descreveram os primeiros casos de esporotricose acometendo seres humanos e ratos.

As espécies do complexo Sporothrix schenckii apresentam como características o dimorfismo e estão amplamente distribuídas na natureza colonizando plantas, árvores e solos em associação com restos vegetais localizados 
principalmente em regiões de clima temperado e tropical úmido (Lopes-Bezerra et al. 2006, Brum et al. 2007).

Apesar de viverem de forma saprófita podem se tornar patogênicos para diversas espécies, existindo relatos em equinos, bovinos, suínos, roedores, primatas, cobras, caninos, felinos e humanos (Macedo \& Costa 1978, Costa et al. 1981, Cheatwood et al. 2003, Meinerz et al. 2008, Souza et al. 2009, Silva et al. 2012, 2013).

Entre os animais domésticos, a esporotricose tem sido frequentemente diagnosticada nos gatos e manifesta-se na forma cutânea localizada, cutânea linfática e cutânea disseminada (Xavier et al. 2004). Em casos graves observam-se manifestações sistêmicas da doença, com comprometimento de diversos órgãos como pulmões, linfonodos internos, fígado, baço e rim (Schubach et al. 2002, Paes 2007).

A infecção nesses animais pode ocorrer pelo contato com o solo a partir do ato de escavar e encobrir os dejetos com terra, por contato com vegetais secos ou em decomposição como locais de afiação ungueal de gatos errantes ou por mordedura ou arranhadura do suscetível por um animal infectado. Esporadicamente existem relatos de transmissão por vias alternativas como a aérea ou digestiva, levando à doença sistêmica (Larsson 2011).

O diagnóstico é realizado através do histórico, sinais clínicos e exames complementares. Os mais empregados rotineiramente por sua praticidade são o cito-diagnóstico e a cultura fúngica. Outros exames laboratoriais mais complexos incluem histotologia, técnica de Imuno-histoquímica e Reação em Cadeia da Polimerase (PCR). Esses exames são mais empregados em pesquisas científicas por exigirem centros de diagnósticos mais sofisticados (Costa et al. 1981, Farias 2000, Rodrigues 2010, Cruz 2013).

Atualmente os felinos apresentam um papel importante como transmissores da esporotricose para humanos, tornando-se um problema de saúde pública no Brasil (Silva et al. 2012, Almeida \& Almeida 2015). Nos últimos anos tem-se observado um aumento no número de animais e humanos infectados, o que vem alterando o perfil da enfermidade, antes predominantemente rural, hoje de ocorrência urbana, tornando-se endêmica, como é o caso do Rio de Janeiro, onde foram confirmados até 2009 cerca de 2.200 casos em humanos em sua maioria adquiridos por arranhaduras ou mordeduras de felinos infectados (Barros et al. 2010, Pires 2012). No nordeste do Brasil apesar da carência de informações, há relatos esporádicos e isolados da doença nos estados do Rio Grande do Norte, Paraíba, Alagoas e Pernambuco (Figueira 2009, Nunes et al. 2011, Marques-Melo et al. 2014, Nogueira 2014).

Esse estudo tem como objetivo relatar um surto de esporotricose felina na Região Metropolitana do Recife.

\section{MATERIAL E MÉTODOS}

Durante o período de março de 2014 a fevereiro de 2016 foram encaminhadas ao Laboratório de Doenças Infecciosas dos Animais Domésticos do Departamento de Medicina Veterinária da Universidade Federal Rural de Pernambuco (UFRPE), 115 amostras de suabes de exsudato de lesões cutâneas, como as demonstradas na Figura 1A, de felinos com suspeita de esporotricose para realização de exame direto e cultura fúngica. As amostras eram provenientes do Hospital Veterinário da UFRPE e de clínicas particulares da cidade do Recife e região metropolitana.

Exame direto. 0 método empregado foi o cito-diagnóstico para confecção de lâminas contendo exsudato das lesões por citologia esfoliativa (impressão e/ou suabe estéril). Em caso de lesões fechadas, o material foi coletado por punção aspirativa por agulha fina. As lâminas foram fixadas e coradas utilizando-se coloração do tipo Romanowsky (Panótico Rápido-Laborclin) para visualização de estruturas celulares e fúngicas (Trabulsi et al. 2002, Larsson 2011).

Cultura fúngica. As amostras coletadas com suabe estéril foram semeadas em Ágar Saboraud acrescido de cloranfenicol e ciclo-heximida, incubadas em aerobiose a $25^{\circ} \mathrm{C}$ durante quatro semanas e a $37^{\circ} \mathrm{C}$ por 3 a 5 dias. Características macroscópicas e microscópicas foram observadas para identificação do fungo (Scott et al. 1996, Brum et al. 2007).

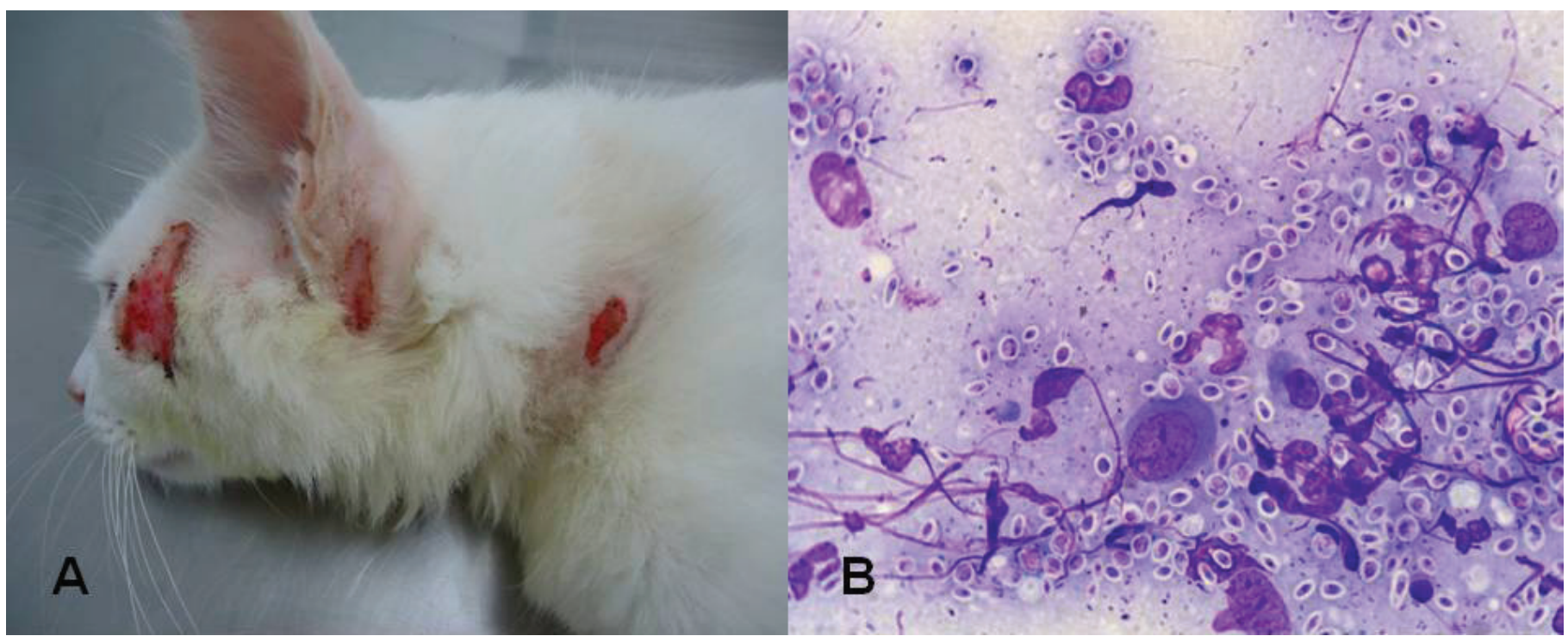

Fig.1. (A) Gato com feridas cutâneas na face, base de orelha e região cervical. (B) Exame direto demonstrando a presença da forma leveduriforme de Sporothrix schenckii. Panótico, obj.10x. 


\section{RESULTADOS E DISCUSSÃO}

Os resultados obtidos encontram-se no Quadro 1. A distribuição dos casos positivos por município de origem do animal está demonstrada no Quadro 2.

Avaliando-se os casos ocorridos entre março de 2014 e fevereiro de 2016 observou-se que das 115 amostras suspeitas, 59 foram positivas para esporotricose, correspondendo a um percentual de 51,3\%. 0 maior número de casos foi registrado no período de 03/2014 a 02/2015. 0 elevado número de atendimento nesse período provavelmente ocorreu devido à falta de informação dos profissionais que desconheciam a doença ou por fracasso na resposta terapêutica ao tratarem os animais para outras enfermidades com sinais clínicos semelhantes. Este fato levou a um aumentou na procura por locais de referência para realização de um diagnóstico mais preciso da doença.

Acredita-se que no período de 03/2015 a 02/2016, a casuística reduziu em consequência do conhecimento adquirido sobre a doença pelos médicos veterinários e tutores por meio de trabalhos de divulgação realizados com a distribuição de panfletos em clínicas veterinárias particulares, postos de saúde e palestras educativas em escolas públicas nos bairros mais acometidos (Nogueira 2014). Outro fator que pode ter influenciado a redução do número de casos registrados da doença pode estar associado ao período de greve ocorrido na Universidade Federal Rural de Pernambuco que se estendeu de maio a setembro de 2015 onde não houve atendimento clínico hospitalar, impossibilitando a coleta e processamento de amostras neste laboratório que também pertence à Universidade o que levou os proprietários a procurar assistência veterinária em clínicas particulares. É importante destacar que este laboratório à época era o único a realizar o diagnóstico desta enfermidade. Como as atividades acadêmicas e de atendimento foram suspensas no período de greve, o diagnóstico não foi solicitado, reduzindo, assim, o registro de casos confirmados da enfermidade neste período. Apesar da redução no número de casos nesse período, acredita-se que a casuística não tenha diminuído, mas tenha sido subnotificada o que dificultou o acompanhamento da disseminação dos casos e a evolução da enfermidade nesta região.

Das 46 amostras positivas no período de $03 / 2014$ a 02/2015, todas foram diagnosticadas ao exame direto e 21 na cultura fúngica. No período de 03/2015 a 02/2016, 13 amostras foram positivas, todas ao exame direto e apenas 6 na cultura fúngica. Nos dois períodos de estudo todas as amostras que tiveram crescimento na cultura fúngica também foram positivas no cito-diagnóstico. Como descrito na literatura e demonstrado na Figura $1 \mathrm{~B}$ ao exame direto foram evidenciadas numerosas leveduras pleomórficas (arredondadas, ovaladas ou em forma de "charuto"), medindo de 2 a $10 \mathrm{~mm}$, circundadas por um halo claro, livres ou fagocitadas (Scott et al. 1996, Hennemann et al. 2003). Outra observação foi o número de leveduras visualizadas que variou de acordo com a gravidade clínica do animal, ou seja, quanto mais grave e disseminada a lesão, maior o número de leveduras encontradas ao exame direto. Os bons resultados obtidos na observação do agente ao exame direto condizem com os relatados por Taboada (2004) que afirma que o cito-diagnóstico é um método simples, rápido, de baixo custo e considerado razoavelmente fácil em felinos. Essa facilidade está diretamente relacionada à espécie felina, pois diferentemente das outras espécies, nesses animais o exsudato presente nas lesões contêm grande quantidade de microrganismos, favorecendo a sua visualização. Essa característica peculiar torna os felinos um importante agente na transmissão do fungo (Larsson 2011, Pires 2012).

No que se refere às culturas fúngicas, estas apresentaram diferentes tempos de crescimento e demonstraram características semelhantes. Macroscopicamente, no início do crescimento foram evidenciadas colônias de coloração creme, escurecendo com a maturidade do cultivo, tornando-se marrom chocolate e posteriormente marrom escuro, quase enegrecida. Na microscopia, observaram-se hifas septadas, com pequenos conídios ovóides nas extremidades sobre um conidióforo, em um padrão lembrando "pétalas de margarida" semelhantes

Quadro 1. Número de amostras analisadas e positivas para esporotricose no exame direto e cultura fúngica

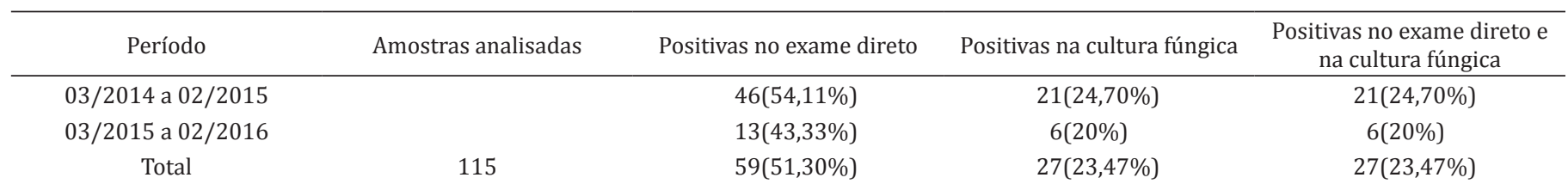

Quadro 2. Número de casos positivos por municípios no primeiro (3/2014 a 2/2015) e segundo $(3 / 2015$ a $2 / 2016)$ período da pesquisa

\begin{tabular}{|c|c|c|c|}
\hline \multirow{2}{*}{ Municípios } & \multirow{2}{*}{ 1ํo Período } & \multirow{2}{*}{ 2º Período } & Total \\
\hline & & & Soma dos períodos \\
\hline Abreu e Lima & 18 & 0 & $18(30,50 \%)$ \\
\hline Igarassu & 3 & 2 & $5(8,47 \%)$ \\
\hline Olinda & 0 & 3 & $3(5,08 \%)$ \\
\hline Paulista & 4 & 2 & $6(10,16 \%)$ \\
\hline Recife & $12^{\mathrm{a}}$ & 3 & $15(25,42 \%)$ \\
\hline
\end{tabular}

a Todos os felinos desse período foram resgatados nas ruas do Recife, não existindo histórico do animal, ${ }^{\text {a }}$ não há registro do local de origem. 
ao descrito por Cruz (2013). Apesar do cultivo negativo em alguns casos deste estudo, os animais foram positivos para esporotricose em decorrência das lesões clínicas típicas, histórico do animal e positividade ao exame direto. Resende \& Franco (2001) relataram que apesar da alta positividade nas culturas fúngicas, os cultivos principalmente em lesões de pele podem ser negativos o que não exclui que seja esporotricose e, por isso, deve-se ser generoso quanto ao número de tubos semeados. Acredita-se que alguns fatores podem influenciar negativamente no isolamento do agente como a quantidade insuficiente de material nos suabes e a contaminação por outros agentes oportunistas que se sobressaem na cultura dificultando o crescimento e identificação do Sporothrix.

Em relação à origem dos animais, no período de $03 / 2014$ a 02/2015 foi constatada a presença de felinos com esporotricose em quatro municípios (Abreu e Lima, Igarassu, Paulista e Recife). No período de 03/2015 a 02/2016, além dos municípios anteriormente citados houve casos registrados no município de Olinda. No primeiro período, os principais municípios foram Abreu e Lima com 18 casos, seguido do Recife com 12, Paulista com 4 e Igarassu com 3. Não há informação sobre a origem dos animais em nove casos. Apesar de o Recife ter o segundo maior número de casos, esses dados são questionáveis, pois todos os casos foram de animais abandonados; desses, 11 foram de felinos abandonados no Hospital Veterinário da UFRPE possivelmente por tutores que achavam que os animais encontrariam tratamento nesta Instituição. Esses casos podem ser oriundos de municípios vizinhos e assim aumentam a casuística do Recife onde está situada a Universidade. Os municípios de Abreu e Lima, Paulista e Igarassu são limítrofes e apresentam características socioeconômicas e ambientais semelhantes, apresentam temperaturas médias entre $25^{\circ} \mathrm{C}-27^{\circ} \mathrm{C}$, umidade relativa acima de $84 \%$ e vegetação predominantemente de Mata Atlântica (IBGE 2016). Essas características ambientais são semelhantes às descritas por Silva (2010) como favoráveis ao desenvolvimento do fungo. Dados epidemiológicos mais consistentes são necessários para explicar os fatores que estão levando a um aumento significativo no número de casos nesses municípios. Pelos registros, os primeiros casos ocorreram em Abreu e Lima seguido de Paulista e Igarassu (Nogueira 2014). Acredita-se que uma das causas da disseminação entre esses municípios seria que após o diagnóstico dos primeiros casos em Abreu e Lima, os proprietários alertados com a possibilidade de contrair a doença estariam abandonando os felinos nos municípios vizinhos.

No segundo período de nossa pesquisa surgem os primeiros casos em felinos comprovadamente residentes em Recife e casos em Olinda. 0 município de Olinda faz limite com Paulista e apresenta características ambientais semelhantes o que provavelmente influencia na ocorrência da doença. Outra informação não oficial é que os animais doentes de Paulista estariam sendo abandonados em Olinda. Ao contrário dos outros municípios, Recife e Olinda possuem características urbanistas, apresentando seu território quase totalmente em área urbana. A forma rápida como ocorreu esse crescimento populacional e sua forma de distribuição no espaço, com desmatamentos, áreas de aglomerados de casas, falta de infraestrutura e saneamento pode predispor às doenças, inclusive, a esporotricose. Esses fatores foram relatados por Silva (2010) que ao estudar a distribuição sócio-espacial da esporotricose humana em pacientes atendidos no Instituto de Pesquisa Clínica Evandro Chagas no período de 1997 a 2007 residentes no Estado do Rio de Janeiro encontrou relação entre a doença, à forma de crescimento populacional e fatores socioeconômicos.

Quanto ao sexo foi observado que a maioria dos animais 46 (78\%) são machos, sendo apenas um castrado e 13 (22\%) são fêmeas. Esses achados estão de acordo com os encontrados por Paula (2008) que afirma que a doença ocorre mais frequentemente em machos jovens em idade reprodutiva. Esse achado pode estar relacionado ao fato dos machos jovens apresentarem maiores episódios de confrontos por disputa territoriais e fêmeas aumentando a chance de inoculação do agente e favorecendo a infecção e a disseminação do fungo (Schubach et al. 2002, Silva et al. 2013). Segundo Larsson (2011), estudos realizados em São Paulo e no Rio de janeiro nos últimos vinte e cinco anos demonstraram que a doença ocorre preponderante em machos (aproximadamente $65 \%$ dos casos).

Foi preconizado o tratamento com iodetos inorgânicos (20 mg/Kg a cada 12 a 24 horas) e itraconazol (5 a 10mg/Kg, por via oral a cada 12 ou 24 horas) para todos os animais positivos. Para evitar complicações medicamentosas foi aconselhado a administração de protetor hepático e acompanhamento mensal dos animais com exame físico, hematológico e bioquímico. Segundo Honse et al. (2010) e Gremião et al. (2006) em casos mais brandos com lesões únicas podem ser realizados métodos alternativos como aplicação de termoterapia local ou incisão cirúrgica com administração de itraconazol, porém, em casos mais graves é comum a ocorrência de recidivas (Schubach et al. 2002, Acosta 2013). Nesse estudo não foi possível o acompanhamento dos felinos durante o tratamento porque os proprietários não retornavam com os animais aos consultórios. Acredita-se que a dificuldade na administração do medicamento, a necessidade muitas vezes de tratamento prolongado, o alto custo da medicação, o receio em adquirir a enfermidade e a dificuldade de deslocamento até o hospital veterinário tenham interferido no retorno dos proprietários.

\section{CONCLUSÕES}

Relata-se o primeiro surto de esporotricose em felinos na região metropolitana do Recife.

Essa doença é uma dermatomicose de importância veterinária que pode causar sérios problemas para felinos, especialmente quando diagnosticada tardiamente e deve ser incluída como diagnóstico diferencial para as dermatopatias ulcerativas nas clínicas veterinárias da região.

Estudos epidemiológicos mais detalhados devem ser realizados para determinar os fatores de risco associados à doença para que medidas de controle possam ser implementadas.

\section{REFERÊNCIAS}

Acosta P.B. 2013. Eficácia da terapia antifúngica na esporotricose felina: relato de casos. Tese de Doutorado, Fundação Educacional Jayme de Altavila, Porto Alegre, RS. 28p.

Almeida L.G.F. \& Almeida V.G.F. 2015. Uma visão interdisciplinar da esporotricose. Revta Eletrôn. Estácio Saúde 4(2).

Barros M.B.L., Schubach T.P., Coll J.O., Gremião I.D., Wanke B. \& Schubach A. 2010. Esporotricose: a evolução e os desafios de uma epidemia. Revta Panam. Salud Publ. 27(6):455-460. 
Brum L.C., Conceição L.G., Ribeiro V.M. \& Haddad Júnior V. 2007. Principais dermatoses zoonóticas de cães e gatos. Clín. Vet. 69:29-46.

Cheatwood J.L., Jacobson E.R., May P.G., Farrell T.M., Homer B.L., Samuelson D.A. \& Kimbrough J.W. 2003. An outbreak of fungal dermatitis and stomatitis in a Free-ranging population of pigmy Rattlesnakes (sistrurus miliarius barbouri) in Florida. J. Wildl. Dis. 39(2):329-337. <http://dx.doi. org/10.7589/0090-3558-39.2.329><PMid:12910760>

Costa E.O., Visintin L.C., Schmidt A.M., Botelho Neto C.A.A. \& Fava Netto C. 1981. Esporotricose-infecção em equinos. Utilização do teste de hipersensibilidade do tipo tardio. Revta Fac. Med. Vet. Zootec. USP 18(1):23-28. <http://dx.doi. org/10.11606/issn.2318-3659.v18i1p23-28>

Cruz L.C.H. 2013. Complexo Sporothrix schenckii. Revisão de parte da literatura e considerações sobre o diagnóstico e a epidemiologia. Vet. Zootec. 20:828. (Edição comemorativa).

Farias M.R. 2000. Avaliação clínica, citopatológica e histopatológica seriada da esporotricose em gatos (Felis catus Linnaeus, 1758) infectados. Dissertação de Mestrado, Faculdade de Medicina Veterinária e Zootecnia, Universidade Estadual Paulista Júlio de Mesquita Filho, Botucatu, SP. 100p.

Figueira K.D. 2009. Esporotricose na espécie canina: Relato de um caso na cidade de Mossoró, RN. Ciênc. Anim. Bras. 10(2):673-677.

Gremião I.D.F., Pereira S.A., Rodrigues A.M., Figueiredo F.B., Nascimento Júnior A., Santos I.B.S. \& Schubach T.M.P. 2006. Tratamento cirúrgico associado á terapia antifúngica convensional na esporotricose felina. Acta Scient. Vet. 34(2):221-223.

Hennemann C.R.A., Guimarães J. \& Bremm M. 2003. Esporotricose felina: uma revisão. Veterinária em Foco 1(1):53-69.

Honse C.O., Rodrigues A.M., Gremiao I.D., Pereira S.A. \& Schubach T.M. 2010. Use of local hyperthermia to treat sporotrichosis in a cat. Vet. Rec. 166(7):208-209. <http://dx.doi.org/10.1136/vr.b4768><PMid:20154314>

IBGE 2016. Estimativas Populacionais do Brasil, Grandes Regiões, Unidades da Federação e Municípios. Metodologia, Instituto Brasileiro de Geografia e Estatística, Pernambuco. Disponível em <http://www.ibge.gov.br/home/> Acesso em 15 jan. 2016.

Larsson C.E. 2011. Esporotricose. Braz. J. Vet. Res. Anim. Sci. 48(3):250-259. <http://dx.doi.org/10.11606/S1413-95962011000300010>

Lopes-Bezerra L.M., Schubach A. \& Costa R.0. 2006. Sporothrix schenckii and Sporotrichosis. Anais Acad. Bras. Ciênc. 78(2):293-308. <http://dx.doi. org/10.1590/S0001-37652006000200009><PMid:16710567>

Lutz A. \& Splendore A. 1907. Sobre uma micose observada em homens e ratos: contribuição para o conhecimento das assim chamadas esporotricoses. Revta Méd. S. Paulo 10(21):443-450.

Macedo M.M. \& Costa E.O. 1978. Estudo da ocorrência da esporotricoseinfecção em animais da espécie bovina. Revta Fac. Med. Vet. Zootec. USP 15(1):059-068. <http://dx.doi.org/10.11606/issn.2318-3659.v15i1p59-68>.

Marques-Melo E.H., Lessa D.F.S. \& Nunes A.C.B.T. 2014. Felino doméstico como agente transmissor de esporotricose para humano: relato do primeiro caso no estado de alagoas. Revta Bahiana Saúde Públ. 38(2):490-498.

Meinerz A.R.M., Antunes T.A., Silva F.V., Xavier M.O., Cleff M.B. \& Meireles M.C.A. 2008. Esporotricose experimental sistêmica em ratos Wistar: avaliação hematológica e perfil hepático. Arq. Bras. Med. Vet. Zootec. 60(4):10261028. <http://dx.doi.org/10.1590/S0102-09352008000400038>
Nogueira A.A. 2014. Esporotricose felina em Pernambuco. Monografia, Universidade Federal Rural de Pernambuco, Recife, PE. 34p.

Nunes G.D.L., Carneiro R.S., Filgueira K.D., Filgueira F.G.F. \& Fernandes T.H.T. 2011. Esporotricose felina no município de Itaporanga, estado da Paraíba, Brasil: relato de um caso. Arq. Ciênc. Vet. Zool. Unipar. Umuarama 14(2):157-161.

Paes R.A. 2007. Antígenos e anticorpos na esporotricose: caracterização e aplicações diagnósticas, Dissertação de Mestrado, Instituto Oswaldo Cruz, Rio de Janeiro. 86p.

Paula R.B. 2008. Esporotricose canina e felina, revisão de literatura. Tese de Doutorado, Universidade Castelo Branco, Rio de Janeiro. 48p.

Pires V.G. 2012. Aspectos antropozoonóticos da esporotricose felina. Tese de Doutorado, especialização em Clínica Médica e Cirúrgica de Pequenos Animais, Universidade Castelo Branco, Rio de Janeiro. 22p.

Resende P.P. \& Franco A.V. 2001. Doenças infecciosas: esporotricose cutâneo linfática. Cad. Bras. Med. 14(1):35-44.

Rodrigues A.M. 2010. Taxonomia polifásica e características proteômicas do complexo Sporothrix schenckii. Dissertação de Mestrado, Universidade Federal de São Paulo, Escola Paulista de Medicina, São Paulo. 240p.

Schubach T.M., de Oliveira Schubach A., dos Reis R.S., Cuzzi-Maya T., Blanco T.C., Monteiro D.F., Barros B.M., Brustein R., Zancopé-Oliveira R.M., Fialho Monteiro P.C. \& Wanke B. 2002. Sporothrix schenckii isolated from domestic cats with and without sporotrichosis in Rio de Janeiro, Brazil. Mycopathologia 153(2):83-86. <http://dx.doi.org/10.1023/A:1014449621732> $<$ PMid:12000130>

Scott D.W., Miller W.A. \& Griffin C. 1996. Dermatologia de Pequenos Animais. $5^{\mathrm{a}}$ ed. Interlivros, Rio de Janeiro. 1130p.

Silva M.A., Medina R.M., Ribeiro R.B., Silveira R.L. \& Carvalho E.C.Q. 2013. Aspectos anatomopatológicos da esporotricose felina. J. Bras. Ciênc. Anim. 6(11):418-426.

Silva M.B.T. 2010. Distribuição sócio-espacial da esporotricose humana de pacientes atendidos no Instituto de Pesquisa Clínica Evandro Chagas no período de 1997 a 2007, residentes no Estado do Rio de Janeiro. Dissertação de Mestrado, Escola Nacional de Saúde Pública Sérgio Arouca, Fiocruz, Rio de Janeiro. 132p.

Silva M.B.T., Costa M.M.M. \& Torres C.C.S. 2012. Esporotricose urbana: epidemia negligenciada no Rio de Janeiro, Brasil. Cad. Saúde Públ., Rio de J., 10(28):1867-1880.

Souza N.T., Nascimento A.C.B.M., Souza J.O.T., Santos F.C.G.C.A. \& Castro R.B. 2009. Esporotricose canina: relato de caso. Arq. Bras. Med. Vet. Zootec. 61(3):572-576. <http://dx.doi.org/10.1590/S0102-09352009000300008>

Taboada J. 2004. Micoses sistêmicas, p.478-503. In: Ettinger S.J. \& Feldman E.C. (Eds), Tratado de Medicina Interna Veterinária: doenças do cão e do gato. $5^{\underline{a}}$ ed. Guanabara Koogan, Rio de Janeiro.

Trabulsi L.R., Alterthum F., Gompertz O.F. \& Candeias J.A.N. 2002. Microbiologia. 3 a ed. Atheneu, Rio de Janeiro. 632p.

Xavier M.O., Nobre M.O., Sampaio Junior D.P., Antunes T.Á., Nascente P.S., Sória F.B.A. \& Meireles M.C.A. 2004. Esporotricose felina com envolvimento humano na cidade de Pelotas, RS, Brasil. Ciência Rural 34(6):1961-1963. <http://dx.doi.org/10.1590/S0103-84782004000600047> 